\title{
Calibrated FEM modelling of rock cutting with PDC cutter
}

\author{
Nina Yari $^{1, *}$, Marcin Kapitaniak $^{1}$, Vahid Vaziri ${ }^{1}$, Lifeng $\mathrm{Ma}^{2}$, and Marian Wiercigroch ${ }^{1}$ \\ ${ }^{1}$ Centre for Applied Dynamics Research, University of Aberdeen, AB24 3UE, Scotland, UK \\ ${ }^{2} \mathrm{~S} \& \mathrm{~V}$ Lab, Department of Engineering Mechanics, Xi'an Jiaotong University, 710049 China
}

\begin{abstract}
Complexity of bit-rock interactions and formation fracture makes rock drilling a challenging task from modelling perspective. A finite element model is developed which can plausibly capture the rock fractures while it is capable of evaluating drilling forces for various values of Rate of Penetration (ROP), bit angular velocity and cutter configuration. Firstly, experiments on a single cutter rig are performed with various drilling parameters to be compared to the proposed FE model. Prior to detailed numerical simulation, a rigorous computation test on material model characterization and the element size and type is conducted to ensure feasible and reliable simulation results. The cutting forces are analysed for different values of WOB, rake angle, initial depth of cut and cutter speed for a single-cutter linear cutting. The formation fragmentation process and computed results are comparable to laboratory tests.
\end{abstract}

\section{Introduction}

Polycrystalline diamond compact (PDC) bits have been used in in drilling industry from early $1970[4,5]$. PDC bits consist of a set of PDC cutters on a tungsten carbide body which shear the rock $[4,5]$. Rock drilling modelling is still a challenge due to the rock material nature and complexity of rock-bit interaction. In order to enhance the efficiency of drilling and improve the drill bit performance, a calibrated model which is able to estimate the drilling forces would be highly beneficial.

In Oil and Gas drilling industry, an axial force is exerted on the bit due to the drill-string weight which is called weight on bit $(W O B)$ while the bit is rotated with an angular velocity $(\omega)$ creating a torque on bit $(T O B)$. In order to investigate drilling forces on each PDC cutter, a linear single cutter rig was designed and built in drilling lab within Centre for Applied Dynamics Research (CADR) at University of Aberdeen. Linear single cutter rig has been used for a long time by many researchers [ 3 , $7,9]$ to model rock-cutter interaction. Similar tests have been performed to assess the unconfined compressive strength of the rock at very small depth of cuts [9].

Numerical simulation methods such as Discrete Element Method (DEM), Finite Element Method (FEM), Finite Difference Method (FEM) and Boundary Element Method (BEM) have been widely used to model rock fracturing and rock-cutter interaction $[6,8]$. The aim of this study is to develop a Finite Element model utilizing ABAQUS CAE which can simulate the rock fracturing and predict the cutting forces in different operating parameters such as the cutter back rake angle, depth of cut and cutter velocity. The model assists in understanding the stress and strain field in the region of rock involved in drilling accompanied by how the cracks are formed and create a chip in the formation. The FE models are then tested against the experimental results.

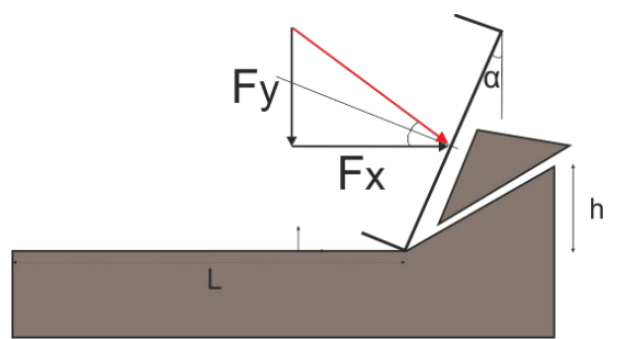

Fig. 1. Linear cutting configuration and the resultant forces.

\section{Experiments}

This section briefly introduces the linear cutting rig followed by test results.

\subsection{Experimental setup}

The linear single cutter rig is a metal shaper machine which is modified to suit for rock cutting. It consists of a stationary table which accommodates the rock, a single PDC cutter (3mm diameter) which is attached to a three dimensional force sensor moving along the rock length to cut a groove. The test is conducted under kinematic control which means the depth of cut and the cutting velocity is constant. Figure 1 represents linear cutting parameters. The cutter velocity in direction of the cutting is equal to $r \omega$, where $r$ is the distance of the cutter from the bit centre. Depth of cut $(h)$ is defined as the bit rate

\footnotetext{
* Corresponding author: nina.yari@abdn.ac.uk
} 
of penetration per revolution $(2 \pi \times R O P / \omega)$. Interfacial frictional angle $(\psi)$ is the angle between the resultant cutting force and the resolved force normal to the cutter [5].

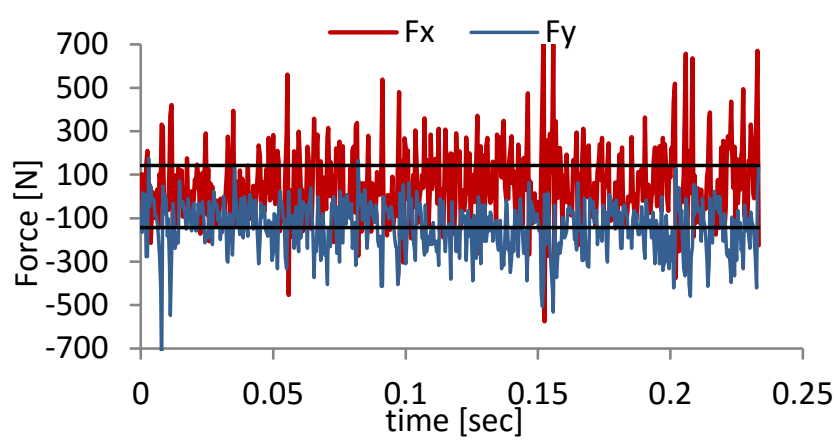

Fig. 2. Experimental results for sandstone cutting with $20^{\circ}$ back rake angle, $1.3 \mathrm{~mm}$ DOC and $500 \mathrm{~mm} / \mathrm{sec}$ cutting velocity

\subsection{Experimental results}

The corresponding cutting and normal force values for the experiment of a $3 \mathrm{~mm}$ single PDC cutter with $20^{\circ}$ back rake angle cutting a groove of $1.3 \mathrm{~mm}$ at $500 \mathrm{~mm} / \mathrm{sec}$ velocity in a sandstone sample are represented in Figure 2. The tests are performed for the same cutter at different values of depth of cut and the resultant cutting forces are measured which are demonstrated in Figure 3.

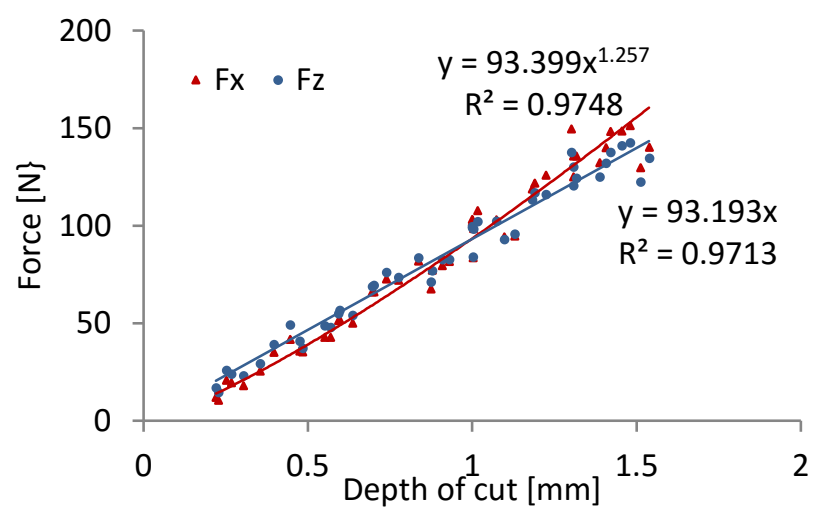

Fig.3. Resultant force values for sandstone with PDC bit with $20^{\circ}$ back rake angle at various depth of cut values.

\section{Numerical models}

Finite Element Code, explicit ABAQUS CAE is utilized for modelling the rock cutting. The rock is modelled as a 2D rectangular deformable solid part and the cutter is a discrete rigid element. The mesh size and type were selected after a careful mesh sensitivity analysis. The bottom part of the rock is fully constrained in all directions while the sides are constrained in $\mathrm{Z}$ direction and the cutter is free to move in X direction. The cutter's size is $3 \mathrm{~mm}$ and its back rake angle is $20^{\circ}$. The contact between the cutter and rock selected as cutter surface to rock node contact to capture the fractures and the friction coefficient is 0.1 .

\subsection{Material model}

Drucker-Prager and Ductile Damage are used as Plasticity and damage models consecutively. DruckerPrager plasticity model represents a pressure dependant yield [1] which makes it a suitable fit for modelling rock plasticity as rock becomes stronger at higher pressure values. Ductile damage is defined as a damage phenomenon by nucleation, growth and coalescence of microscopic voids [1]. In microscopic scale, the rock consists of voids and the cracks are formed by similar phenomenon. In Ductile Damage criterion, the equivalent plastic strain at the damage initiation is a function of strain rate and stress triaxiality $\eta$, which is the ratio of mean stress to Von-Mises stress [1]:

$$
\omega_{D}=\int \frac{d \bar{\varepsilon}^{p l}}{\bar{\varepsilon}_{D}^{p l}\left(\eta, \dot{\varepsilon}^{p l}\right)}=1
$$

Where $\bar{\varepsilon} p l$ is equivalent plastic strain at the onset of damage and $\dot{\overline{\varepsilon^{p}} l}$ is its rate and $\omega_{D}$ is the damage parameter which increases with plastic deformation [1]. As soon as the damage initiated, the stiffness is degraded which is identified by damage evolution law. In ABAQUS, the damage evolution is governed by [1]:

$$
\sigma=(1-D) \bar{\sigma}
$$

Where $\mathrm{D}$ is damage variable, $\sigma$ is stress tensor at any time and $\bar{\sigma}$ is undamaged stress tensor. Published data on triaxial experiments on Vosges Sandstone [2] along with some experimental results were used as material model parameters.

\subsection{Mesh size sensitivity analysis}

The results of FE models are highly sensitive to mesh size and type. After a careful analysis, it is found that triangular elements are better representative of rock heterogeneity compared to quadrilateral elements. In order to select element size for rock, the model was run with various numbers of elements and the resultant force values were compared to each other (Figure 4). As displayed in Figure 4, the results cutting force value is converged when 48542 elements are used where the size of each element in cutting zone is $0.05 \mathrm{~mm}$. As the high stress region in rock cutting is at the vicinity of the contact, the mentioned element size is used in top part of the formation where cutting takes place and coarser elements forms the rest of the rock.

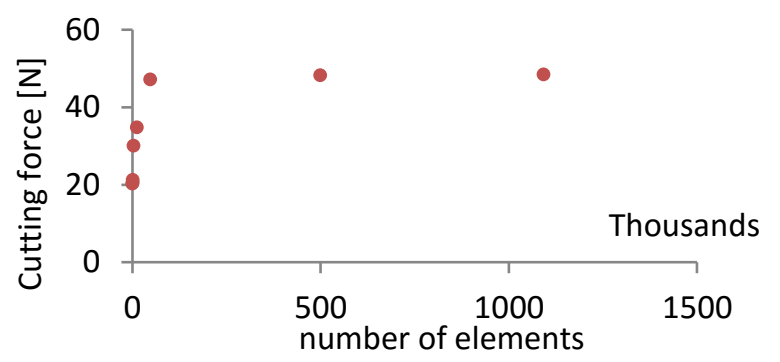

Fig. 4. Cutting forces from FE model for different rock element numbers 


\subsection{Numerical model results}

\subsubsection{Fixed depth of cut}

Figure 5 shows the stress field of the FE model when a $3 \mathrm{~mm}$ cutter with $20^{\circ}$ back rake angle is cutting a groove of $1.4 \mathrm{~mm}$ along the rock with $500 \mathrm{~mm} / \mathrm{sec}$ velocity. The force responses of the numerical simulation for the same cutter with $0.7 \mathrm{~mm}$ depth of cut are demonstrated in Figure 6 .
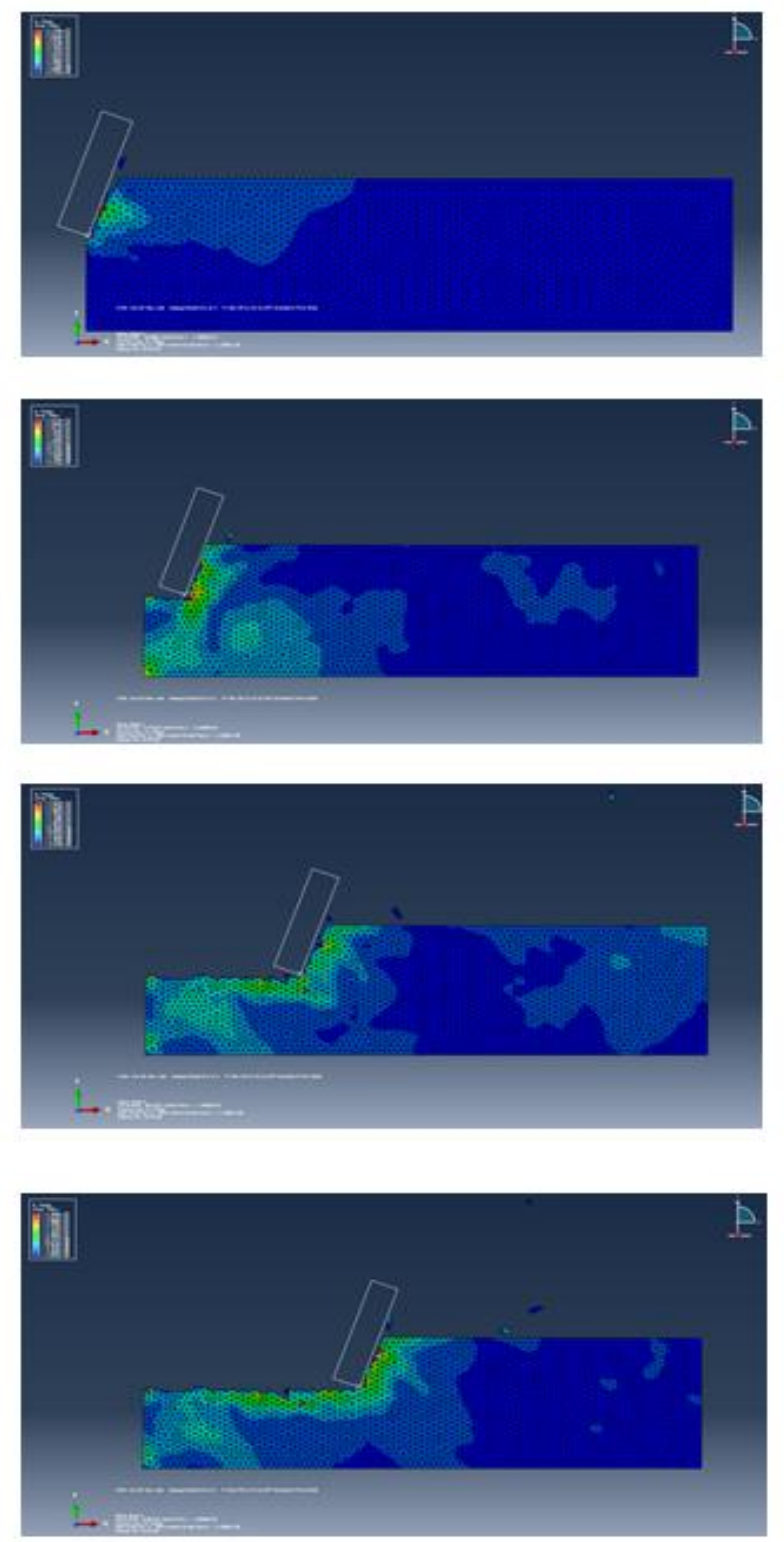

Fig. 5. Stress field evolution for a $2 \mathrm{D}$ FEM model of rock cutting with a single cutter for cutting velocity of $500 \mathrm{~mm} / \mathrm{sec}$, $20^{\circ}$ back rake angle and $1.4 \mathrm{~mm}$ depth of cut

\subsubsection{Unfixed depth of cut}

In real drilling condition depth of cut is not fixed throughout the cutting process and it is increasing by time. As a result, there would be a velocity in $\mathrm{Z}$ direction as well. Consequently, work done $(W)$, volume of cut $(V)$, cutting velocities $\left(v_{x} \& v_{y}\right)$ and arear of cut $(A)$ are calculated as:

$$
\begin{gathered}
W=F_{x} d l+F_{z} d h \\
V=A_{\text {cutting }} \times L \\
v_{x}=\frac{d l}{d t}, v_{z}=\frac{d h}{d t}
\end{gathered}
$$

$$
A_{\text {cutting }}=\left[\arccos \left(\frac{r-h}{r}\right) r^{2}-\sqrt{r^{2}-(r-h)^{2}}(r-h)\right]
$$

Where $h$ is depth of cut and $L$ is the length of the rock in $X$ direction.

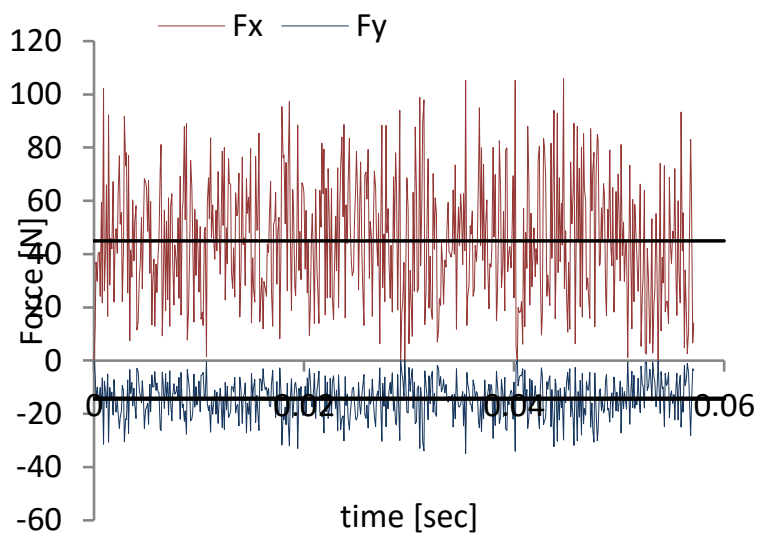

Fig. 6. Computer cutting force values for sandstone with PDC bit with $20^{\circ}$ back rake angle, $0.7 \mathrm{~mm}$ depth of cut and $500 \mathrm{~mm} / \mathrm{sec}$ cutting velocity

Assuming that cutting efficiency $(\eta)$ is a parameter equal to:

$$
\eta=\frac{\text { cutting volume rate }}{\text { work rate }}
$$

So:

$\eta=\frac{d V / d t}{d w / d t}=\frac{d V}{d w}=f\left(r, v_{x}, v_{z}\right)=g\left(r, \frac{v_{z}}{v_{x}}\right)=g\left(r, \frac{d h}{d l}\right)$

Because $r$ is fixed:

$$
\eta=\eta\left(\frac{d h}{d l}\right)
$$

Consequently, at a ratio of horizontal velocity to vertical velocity, the efficiency of drilling is optimum.

The model was run for various quantities of velocity ratios and Figure 7 is an example of the stress field and computed force response when $\mathrm{v}_{\mathrm{z}} / \mathrm{v}_{\mathrm{x}}$ is 0.2 .

Figures 8 and 9 demonstrate FEM computed forces and efficiencies for various velocity ratios. It is shown that for each test condition, there is an optimum velocity ratio which enhances the cutting efficacy. 
(a)

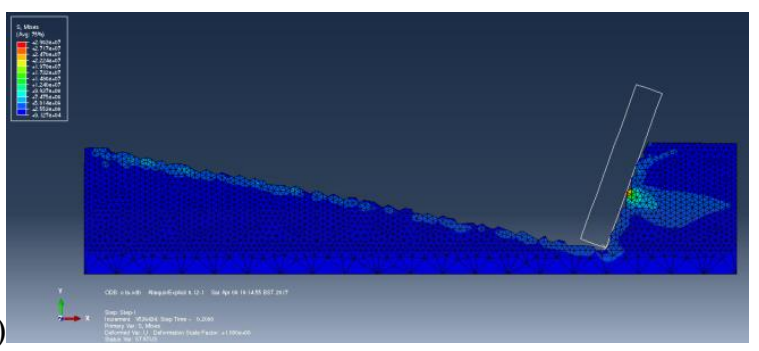

(b)

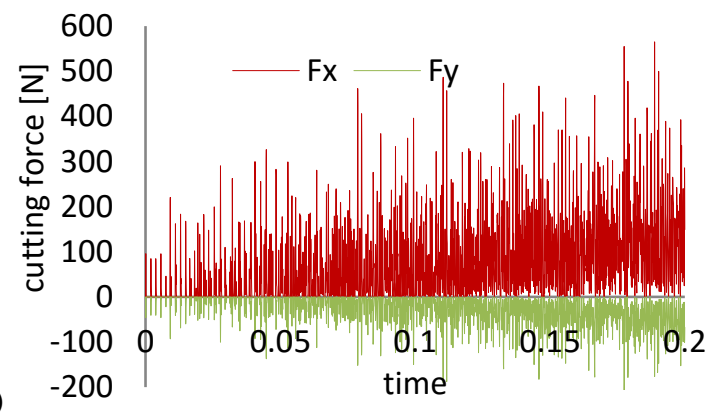

Fig. 7. (a) Stress field 2D FEM model of rock cutting with a $3 \mathrm{~mm}$ single cutter with $20^{\circ}$ back rake when $\mathrm{v}_{\mathrm{x}}=400 \mathrm{~mm} / \mathrm{sec}$ and $\mathrm{V}_{\mathrm{z}}=80 \mathrm{~mm} / \mathrm{sec}$, (b) computed cutting force vs cutter advance

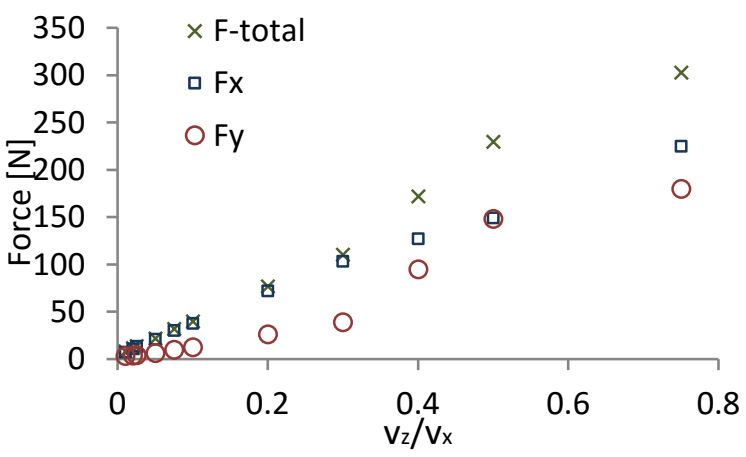

Fig. 8. Computed cutting forces for $2 \mathrm{D}$ FEM model of rock cutting with a $3 \mathrm{~mm}$ single cutter with $20^{\circ}$ back rake for unfixed depth of cut vs velocity ratio

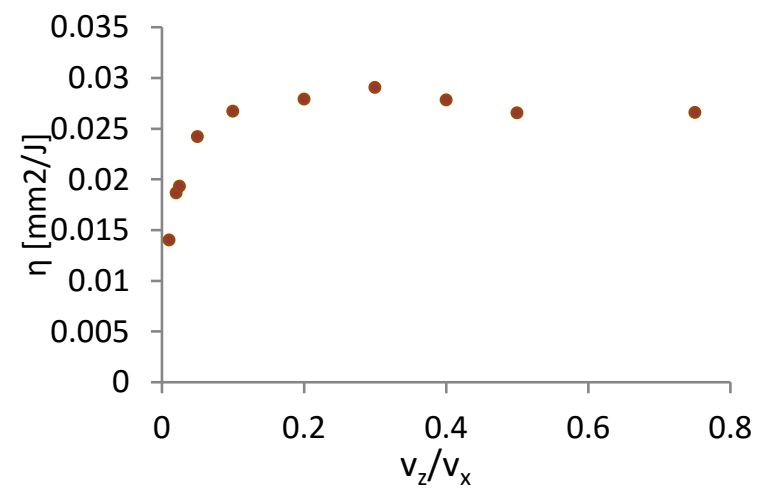

Fig. 9. Cutting efficiency vs velocity ratio for 2D FEM model of rock cutting with a $3 \mathrm{~mm}$ single cutter with $20^{\circ}$ back rake for unfixed depth of cut

\section{Conclusion}

In this study, a drilling process has been studied with both experimental and numerical simulations. To compare to the proposed numerical model, experiments on a single cutter rig have been first performed with various drilling parameters. Then a finite element model has been developed, and the cutting forces have been analysed for different values of WOB, rake angle, initial depth of cut and cutter speed for a single-cutter linear cutting. To evaluate the drilling process, a new parameter, the drilling efficiency, has been proposed. Initial numerical simulations demonstrated that this parameter can be employed to optimize the drilling parameters. The proposed parameter will be analysed further by being used for complex drilling.

\section{References}

1. ABAQUS documentation. USA: Dassault Systems.

2. P. Bésuelle, J. Desrues, S. Raynaud, Experimental characterisation of the localisation phenomenon inside a vosges sandstone in a triaxial cell, Int. J. Rock Mech. Min. Sci., 37 (2000)

3. D. Che, W.L. Zhu, K.F. Ehmann, Chipping and crushing mechanisms in orthogonal rock cutting, Int. J. Mech. Sci., 119 (2016)

4. E. Detournay, P. Defourny, A phenomenological model for the drilling action of drag bits, (1992)

5. E. Detournay, T. Richard, M. Shepherd, Drilling response of drag bits: Theory and experiment, Int. J. Mech. Sci., (2008)

6. M. C. Jamie, Y. Zhou, J. Kin, I. K. Gamwo, Finite element modelling of rock cutting and its fragmentation process, Int. J. Rock Mech. Min. Sci., 80 (2015)

7. Y. Nishimatsu, The mechanics of rock cutting, International Journal of Rock Mechanics and Mining Sciences and Geomechanics Abstracts, (1972)

8. L. M. Pradeep, Influence of cutter velocity, friction coefficient and rake angle on the formation of discontinuous rock fragments during rock cutting process, Int. J. Adv. Manuf. Tech., (2016)

9. T. Richard, F. Dargain, E. Poyol, E. Detournay, Rock strength determination from scratch tests, Eng. Geol., (2012) 